\title{
Internet of Things: Application and Prospect
}

\author{
Ran Liu $^{1}$ and Jinfeng Wang ${ }^{1}$ \\ ${ }^{1}$ School of Management Engineering,Zhengzhou University,Zhengzhou Henan 450001, China
}

\begin{abstract}
Nowadays, Internet of Things (IOT) is growing rapidly, which is used to the extension of the Internet and the Web into the physical realm, by means of the widespread deployment of spatially distributed devices with embedded identification, sensing and actuation capabilities. Internet-of-Things envisions a future in which digital and physical entities can be linked, by means of appropriate information and communication technologies, to enable a whole new class of applications and services. In this paper, the origin and concept of the Internet of things are introduced, and then the potential application areas and the impact of the Internet of things are analyzed.
\end{abstract}

Keywords. Internet of Things, Application research.

\section{Introduction}

Now, about 2 billion people around the world use the Internet to browse web pages, send and receive messages, use social networking applications and or do the other activities. With the development of the Internet information technology, Internet of things (IOT) has become a new round of economic and technological development, one of the strategic high ground in the world. In the information industry field, whether engineers engaged in the information technology industry consulting and research of the company's or consumers of the information technology products, are optimistic about the prospects of the Internet of things. From Google to apple, Microsoft, more and more IOT related products appeared in the current industry, all of them investing a large amount of capital and manpower in the field of IOT to develop the consumers favorite products, hope to lead the development of the industry [1]. IOT as a global platform for physical entities and intelligent devices to communicate, calculate and coordinate, It is foreseeable that the deep integration of IOT technology and other industries can change the mode of economic development, achieve the real green growth and low-carbon economy. The IOT economy will lead the future of sustainable economic and social development.

The goal of this paper is to study the Internet of Things with a new angle, including the concept and application field of IOT. In fact, research topics related to IOT topics are still highly fragmented and largely focused on single application domains or single technologies. In addition, participation in the field of networking and communication science is still limited, although their contribution to the development of the field has great potential. The main contents of this paper are as follows: Firstly, introduced the main concepts of Internet of Things. Then the potential applications and impact areas of IOT are analyzed. Finally, the research contents of this paper are summarized.

\section{Origin and definition}

\subsection{Origin}


The concept of IOT in 1999, by the Massachusetts Institute of Technology Auto-ID Lab, the first idea of the IOT is using Radio Frequency Identification (RFID), encoding and Internet technology to establish a kind of global information sharing of the "Internet of Things". From its name can know that IOT are connected the Internet with things. There are two meanings: First, the core technology of things is still the Internet, is the extension and expansion of the Internet. Second, the client extends and expansions between the objects and objects, so as to carry out information exchange and communication. IOT through the intelligent perception, identification technology, pervasive computing and other communication-aware technology integrates into the Internet widely. Application characteristics is the core of IOT, user experience and innovation is the soul of the development of IOT [2]. In 2003, the United States, "Technology Review" propose the point that "sensor network technology will change the people's way of life in the future as the top ten technology." In 2005, the International Telecommunication Union (ITU) formally defined the concept of IOT, published "ITU Internet Report 2005: Internet of things", and describes the form of IOT, features, technologies, opportunities and challenges. With the development of science and technological progress, the concept of IOT is also expanding. The current IOT can achieve a comprehensive sense of things, identification and control, through the wireless connection to the network, intelligent reasoning and decision-making.

\subsection{Definition}

Sun Qibo [3] puts forward the concept of Internet of things from the definition origin, development, features. From a narrow sense, things are connected to multiple items of the network, and these items can be identified and managed. The broad sense of things can be considered to be a fusion of the physical world and information world, everything will be networked, digital. Achieve efficient information exchange between the environment and people, objects and objects, people and objects. the information technology will be into our society deep-dyed.

The Internet of things white paper, "the Internet of things is the extension of network's application and the Internet, it uses sensing technology and intelligent device for perception to sense the physical world. Calculating by Internet, process and knowledge mining to realize information exchange between people and objects, objects and objects, so as to seamless links to physics the real-time control, to accurate management and scientific decision-making [4]."

Zhou Hongbo [5] and other scholars believe that the concept of the definition refers to the terminal equipment and facilities everywhere, through a variety of wireless or wired, short or long distance to achieve interoperability ,application integration, and cloud computing operating mode based on network, Internet environment. Using appropriate security mechanisms to provide safe and controllable personalized location tracing, alarm linkage, dispatching plan management, remote control, security, remote maintenance, online upgrade, statistics, decision support, leadership desktop management and service functions, to achieve high efficient, energy saving, safety, environmental protection tube control, business integration."

Zhu Hongbo [6] that IOT is a name with the network will combine things together. IOT is currently define as a net that making use of radio frequency identification (RFID), infrared sensors, GPS, laser scanners and other information sensing equipment, and according to the agreement, the goods connect with the network to realize the exchange of information and communication, intelligent identification, positioning, tracking, monitoring and management of a network.

IOT use local network and Internet communication technology such as sensors, controllers, machines to realize communication between people and objects, objects and objects by a new contact links .So as to achieve information resources, Remote program control and intelligent control network [2].

\section{Application and prospect}

IOT has a great potential for development in almost all areas, which mainly due to the Internet of things can be context-aware (for example, can collect information on natural parameters, medical parameters, or user habits), and provide tailored services. Regardless of the application area, the purpose of these applications is to improve the quality 
of people's everyday life and will have a profound impact on the economy and society. The application of IOT can be divided into three areas: industrial areas, smart city areas and health areas. Each domain is not separate but partially overlapping, because some applications are shared. For example, product tracking, usually in the industrial and health fields, can be used to monitor goods or food, but it can also be used to monitor the distribution of medicines.

Table 1. The main application areas of Internet of things.

\begin{tabular}{|c|c|c|}
\hline $\begin{array}{c}\text { Main Application } \\
\text { Areas }\end{array}$ & Domain Segmentation & Examples \\
\hline \multirow{6}{*}{ Industry } & \multirow{2}{*}{$\begin{array}{c}\text { Logistics and Product Lifecycle } \\
\text { Management }\end{array}$} & Inventory management, cargo transport \\
\hline & & Food preservation \\
\hline & \multirow{2}{*}{$\begin{array}{l}\text { Agriculture and animal } \\
\text { husbandry }\end{array}$} & Agricultural Production and Breeding \\
\hline & & Animal Disease Infection Control \\
\hline & \multirow{2}{*}{ Industrial processes } & Real - time vehicle diagnosis \\
\hline & & Baggage Management \\
\hline \multirow{4}{*}{ Smart city } & \multirow[b]{2}{*}{ Smart Home / Building } & Home Area Network (HAN) \\
\hline & & $\begin{array}{c}\text { Multimedia management, power } \\
\text { management }\end{array}$ \\
\hline & \multirow{2}{*}{$\begin{array}{c}\text { Public Safety and } \\
\text { Environmental Monitoring }\end{array}$} & Environmental monitoring \\
\hline & & Smart mobile devices \\
\hline \multirow{4}{*}{ Health Care } & \multirow{2}{*}{ Disease diagnosis and treatment } & Real-time chiri-cal consmlfation \\
\hline & & Vital signs monitoring \\
\hline & \multirow{2}{*}{ Independent live } & Assisted persons with disabilities \\
\hline & & emergency rescue \\
\hline
\end{tabular}

Table 1 shows the breakdown of the above three application areas of the IOT. It is noteworthy that not all of the IOT applications have the same level of maturity. Some of these applications, especially for the user is the simplest and most intuitive application, is already part of our daily lives. But many are still in the experimental stage, because they need to work with different users for better cooperation. Finally, there are many applications in its infancy. The remainder of this section provides a detailed introduction to the various areas of application.

\subsection{Industry}

IOT can be applied to all industrial activities, including inter-firm commercial or financial transactions, organizations and other entities, mostly used in logistics, manufacturing, regulatory processes, services, banking, intermediaries and so on.

\subsubsection{Logistics and product lifecycle management}

A related example of industrial applications is logistics and supply chain management. Electronic tags attached to specific objects can be used to identify the type of material and commodity that can be clothing, furniture, equipment, food or liquids [7]. The use of electronic tags contributes to effectively manage warehouses and retail and reduce inventory through accurate knowledge of the current inventory, while reducing inventory uncertainties. The entire lifecycle of the goods can also be tracked [8], for example, a RFID reader installed at a manufacturing facility can monitor the production process, and the label can be traced back to the entire supply chain (e.g., packaging, transportation, warehousing). An advanced IOT system consisting of RFID equipment and smart shelf real-time tracking equipment can help to reduce material waste, thereby reducing costs and improving margins for retailers and 
manufacturers. For example, if the shelf keeps some free items, sales are reduced by approximately $8.3 \%$ [9]. If there is a correct estimate of what is needed, the shortfall and overproduction can be substantially reduced, which can be inferred from the data collected on the smart shelf. In addition, the real-time analysis of the sensor can determine the deterioration of the product, which is essential to food and liquid. For example, to ensure continuous monitoring of the temperature and humidity in a warehouse or cold store for fresh perishable goods such as fruit, vegetables, frozen food, the actuator can adjust the relevant parameters to best ensure the quality of the food.

\subsubsection{Agriculture and animal husbandry}

IOT contributes to agriculture and breeding. Actually, animal traceability provisions require continuous monitoring of animals and their migration in order to report any relevant events, such as disease, to the authorities in a timely and appropriate manner. The use of IOT identification systems (e.g., radio frequency identification, sensors) allows the identification and monitoring of animals and isolates infected animals from healthy animals [10], thereby avoiding the spread of infectious diseases. Advanced chips can store information about the physical condition of animals (e.g., quantity information, veterinary examination, disease infection, vaccination) or send information about the health of animals(e.g., temperature) to simplify animal health certificates, control trade and imports, and avoid possible fraud. By analyzing the data collected, the authorities can verify the actual number of livestock reported by the local breeder and provide subsidies. The use of advanced sensor systems to monitor and control agricultural production and feed (such as additives, pigments) is a further application of the Internet of Things.

\subsubsection{Industrial processes}

IOT can provide advanced solutions for the automotive industry. Real-time vehicle diagnosis is a key application, everything can be monitored by a specific sensor: tire pressure, motor data, fuel consumption, position, speed, distance from other vehicles, etc., and then report the sensed data to the central system [11]. The wireless identification technology attached to the vehicle component can record the history of a particular motor moving part and improve the assembly process by automatically finding missing parts. The application of IOT technology makes people and goods transportation system more advanced, such as ticket pricing, more secure baggage management based on automatic tracking and sorting. The industrial management system based on IOT technology can monitor the industrial plant, for example, to reduce the incidence of accidents, especially in high-risk areas (e.g., oil and gas plants), sensors connected to containers for the transport of dangerous goods may send different signals indicating the chemical composition of the cargo and the composition Of the highest level. In the case of critical situations (e.g., where a chemical composition is approaching the maximum allowed level in a particular geographical area), the sensor can automatically send an alarm to the control center.

\subsection{The area of smart city}

IOT will help to improve the environmental sustainability and the life quality of the people in our country. The key point is the energy and how to use the resources efficiently, and seeking intelligent solutions to enjoy personal life

\subsubsection{Smart home / building}

Integrating communication technology into building and housing, the future smart city building or home will be equipped with a variety of sensors and smart devices (e.g., broadband gateway, cell phone, computer, TV, surveillance camera and lamplight). Some application programs use the simplest functions in the IOT, for example, the program to ensure the safety (e.g., video surveillance and intrusion detection), plant management and maintenance (e.g., fault detection), service Automation (e.g., HVAC and lighting ), and entertainment system (e.g., multimedia distribution at home). Other types of applications integrate smart grid, and optimize the domestic consumption situation at the same 
time [12]. For example, the home area network (HAN) allows the electrical appliances to interact with the intelligent instrument, and ensures the required performance while reduces the cost. It can also arrange a variety of home appliances with intelligent work to avoid the peak period. People can see the using situation of home appliances and other equipment through the smart phone anywhere and anytime, monitor the running state of the system intelligently, and control home appliances at a specified time to open, close or set running parameters to make the family environment more suitable for family members to live. For example, through the analysis of information flow, the system can understand the time to arrive home of a person, open the door and light, and fill the bathtub with water. Users can change or cancel the automatic operation at any time.

\subsubsection{Public safety and environment monitoring}

Public safety services include the maintenance of public order, the prevention and protection of citizens, and the protection of public and private property. Emergency management helps the society to prevent and respond to natural or man-made disasters, such as chemical leakage, water flood, conflagration, epidemic disease and power interruption. The IOT provides solutions to monitor and track these emergencies [13]. Collecting data from a fixed camera and personal equipment located within the city can implement advanced video surveillance and regional monitoring, and help the police in holding sports events, music performances, political meetings to maintain public order at the same time. Private and public building security (e.g., banks, stores) can be enhanced by using the sensor technology to trigger the alarm, emergency action can also be improved and strengthened. At present, the emergency system is short of accurate information about the scene of the emergency. Dedicated sensors and smart cameras, as well as global positioning system (GPS) providing real-time positioning and tracking of and wireless technology, can be used to outline the adumbration of an event, forecast the trend of events (e.g., the direction and speed of fire spread) so as to establish a dynamic emergency planning to coordinate the rescue operation.

\subsection{Health care}

IOT will play an important role in the development of intelligent services to improve people and social activities. IOT that involve citizens and communities in governance and government decision-making (for example, online political participation) to allow people to live independently or to maintain their social relationships, improve health and social care.

\subsubsection{Disease diagnosis and treatment}

The healthcare industries will be strongly influenced by IOT. Advanced sensing devices can monitor patient vital signs (e.g., body temperature, blood pressure, heart rate, and cholesterol levels) in real time and then transmit the acquired data to a doctor via a specific communication technique (e.g., Bluetooth, Wireless HART, ISA100) for diagnosis and monitoring of the patient's signs. Body area networks (BANs) are interconnected through wearable devices that allow remote monitoring of patient status outside the hospital [14]. Internet of things can also be used in the identification of drugs and medical equipment identification, for example, the use of smart labels will ensure that items are accurately tracked to prevent loss or theft, or items in a surgical procedure to be left in the patient (e.g., gauze or other small items). Smart tags are also important for the inventory management of medical devices.

\subsubsection{Independent live}

IOT has many advantages that can improve the quality of people's live. Wearable sensors if connect to personal computer devices and run specific applications can allow people to record their daily activities (walking distance, calorie expenditure, exercise conditions, etc.), providing many ways for them to improve their lifestyles and prevent health problems. IOT can be added through the sensor device to monitor the physical state of the elderly. By monitoring 
the sensor's real-time acquisition of physiological status information, the system will be able to set up a medical alert, advise on hospitalization. It is also possible to diagnose early Alzheimer's disease by observing abnormalities in normal life. The mobile assistant is also a basic service that allows the elderly to move safely in the city or to use public transport. Through the data collected by the personal mobile device (equipped with sensors such as position sensors, direction sensors, motion obstacle detection sensors, cameras) and the fixed sensor in the city, the mobile phone artificial intelligence system can perceive the surrounding environment and transmit the related information to the user through the synthesized speech. Similar applications can help visually impaired people improve their ability to move around the city [15].

\section{Conclusion}

IOT may represent the next leap forward in the information and communications technology field. The large-scale deployment of embedded applications increases the possibility of a real-world and virtual world to merge seamlessly, opening up new research and business directions.

This article provided an overview of the key issues related to the development of IOT technologies and services. The most relevant application fields have been presented. We do hope that this survey will be useful for researchers and practitioners in the field, helping them to understand the huge potential of IOT. Although we have outlined the main applications of Internet of things, but The challenges that IOT may be faced in practical applications of the future may not be covered in this paper, future research can proceed from this aspect.

\section{References}

1. J. Ma, X. Zhou, S. Li, Z. Lio, Connecting agriculture to the internet of things through sensor networks, in: Proceedings of Internet of Things (iThings/CPSCom), 2011, pp. 184-187.

2. Liu Shuncai. Discussion on the development of the Internet of things technology and its application of network security technology and application, 2016,04:93-94.

3. Sun Qibo, Liu Jie, Li Shan, Fan Chunxiao, Sun Juanjuan. The Internet of things: concept, architecture and key technology research. Journal of Beijing University of Posts and Telecommunications, 2010,03:1-9.

4. Zhou Jin. The basic theory and key technology of information fusion in the Internet of things. Jilin University, 2014.

5. Internet of things white paper (2011). China Public Security (integrated version), 2012, Z1:138-143.

6. Zhu Hongbo, Yang Longxiang,. Research on the technology and application of Internet of things. Journal of communication, 2010,11:2-9.

7. F. Villanueva, D. Villa, F. Moya, M. Santofimia, J. Lopez, Internet of things architecture for an RFID-based product tracking business model, in: Proceedings of 6th Innovative Mobile and Internet Services in Ubiquitous Computing (IMIS), 2012, pp. 811-816.

8. H. Cai, L.D. Xu, B. Xu, C. Xie, S. Qin, L. Jiang, IoT-based configurable information service platform for product lifecycle management, IEEE Trans. Indust. Inform. 10 (2) (2014) 1558-1567.

9. T.W. Gruen, D.S. Corsten, S. Bharadwaj, Retail Out-of-Stocks: A Worldwide Examination of Extent, Causes and Consumer Responses, Tech. rep, 2002.

10. A.S. Voulodimos, C.Z. Patrikakis, A.B. Sideridis, V.A. Ntafis, E.M. Xylouri, A complete farm management system based on animal identification using RFID technology, Comp. Electron. Agricult. 70 (2) (2010) 380-388.

11. P. Hank, S. Müller, O. Vermesan, J. Van Den Keybus, Automotive ethernet: in-vehicle networking and smart mobility, in: Proceedings of the Conference onDesign, Automation and Test in Europe (DATE'13), 2013, pp. $1735-1739$. 
12. J. Lu, T. Sookoor, V. Srinivasan, G. Gao, B. Holben, J. Stankovic, E. Field, K. Whitehouse, The smart thermostat: using occupancy sensors to save energyin homes, in: Proceedings of the 8th ACM Conference on EmbeddedNetworked Sensor Systems (SenSys '10), 2010, pp. 211-224.

13. E. Sun, X. Zhang, Z. Li, The internet of things (IOT) and cloud computing (CC)based tailings dam monitoring and pre-alarm system in mines, Safety Sci. 50(4) (2012) 811-815.

14. F. Delmastro, Pervasive communications in healthcare, Comp. Commun. 35(11) (2012) 1284-1295.

15. R. Manduchi, J. Coughlan, (computer) vision without sight, Commun. ACM 55(1) (2012) 96-104. 